\title{
Exact Solutions of a Fermion-Soliton System in Two Dimensions
}

\author{
L. Shahkarami* and S.S. Gousheh \\ Department of Physics, Shahid Beheshti University G.C., Evin, Tehran 19839, Iran
}

September 10, 2018

\begin{abstract}
We investigate a coupled system of a Dirac particle and a pseudoscalar field in the form of a soliton in $(1+1)$ dimensions and find some of its exact solutions numerically. We solve the coupled set of equations self-consistently and non-perturbatively by the use of a numerical method and obtain the bound states of the fermion and the shape of the soliton. That is the shape of the static soliton in this problem is not prescribed and is determined by the equations themselves. This work goes beyond the perturbation theory in which the back reaction of the fermion on soliton is its first order correction. We compare our results to those of an exactly solvable model in which the soliton is prescribed. We show that, as expected, the total energy of our system is lower than the prescribed one. We also compute non-perturbatively the vacuum polarization of the fermion induced by the presence of the soliton and display the results. Moreover, we compute the soliton mass as a function of the boson and fermion masses and find that the results are consistent with Skyrme's phenomenological conjecture. Finally, we show that for fixed values of the parameters, the shape of the soliton obtained from our exact solutions depends slightly on the fermionic state to which it is coupled. However, the exact shape of the soliton is always very close to the isolated kink.
\end{abstract}

\section{Introduction}

Systems consisting of coupled fermionic and bosonic fields, specially when the latter is in the form of a soliton or a solitary wave, have played an important role in many branches of physics. Since solitons are non-dispersive localized packets of energy, they present the possibility of describing extended objects, such as hadrons as stable states within quantum field theory. For example, starting with the work of Skyrme [1, 2, 3, 4, 5] in 1958, much work has been done for describing hadrons and their interactions in the non-perturbative QCD regime using phenomenological nonlinear field theories. The simplest examples of such models are the variety of bag models $[\underline{6}, \mathbf{7}, \mathbf{8}$, 9, 10, 11, 12, 13 in some of which the effect of gluons is replaced by the interaction of scalar fields with the quarks [14, 15]. For example static bag models such as MIT and SLAC models have had notable success in describing hadronic structure. In the papers on the SLAC bag model, first proposed by [9], several hadronic properties including their quantum numbers, form factors, as well as the bound-state energies are calculated.

A coupled fermion-soliton system also appears in a set of works that examine the effects of quantum corrections on solitons and the formation of a quantum soliton which is not present at the classical level [16, 17, 18, 19, 20]. In these works, the authors consider a theory of a scalar field coupled to a heavy fermion, i.e. a strongly coupled fermion-scalar system. In these

*Electronic address: l_shahkarami@sbu.ac.ir 
models fermions acquire mass through their couplings to a scalar field with non-vanishing vacuum expectation value. According to this assertion, a strong fermion-scalar coupling is analogous to the existence of heavy fermions. Considering the zero and first order quantum corrections, they showed that heavy fermions can stabilize the soliton.

Fermion-soliton systems also appear in the context of the so-called braneworld models. A major issue in these models is the localization problem [21, 22, 23] of the Standard Model fields on the brane. Localizing a fermion on the brane was first described by the original work of Rubakov and Shaposhnikov [24], in which a 5-dimensional model, i.e. one with codimension-1, was employed and the brane Lagrangian was the $\phi^{4}$ (or sine-Gordon) system in one dimension, supporting the kink soliton. Extension to higher dimensions for this mechanism has been performed by other authors [25, 26, 27, 28]. In the following years, there has been some considerable activities in this context [29, 30, 31].

A more curious phenomenon when a fermion interacts with a soliton is the assignment of half-integer fermion number to the solitonic states. This was first pointed out by Jackiw and Rebbi [32. They considered the structure of a coupled fermion-soliton system which possesses $\mathrm{CP}$ symmetry and showed that the existence of a zero-energy fermion mode, implies that the soliton is a degenerate doublet carrying fermion number $\pm \frac{1}{2}$. The consequences of this surprising result have been studied extensively for many different physical phenomena in the literature, such as in the context of condensed-matter systems [33, 34, polyacetylene being the standard example [35, 36, and in high energy physics. Goldstone and Wilczek [37] introduced a powerful method, called the adiabatic method, for calculating the vacuum polarization of the Fermi field induced by the soliton. In this method, the topologically non-trivial configuration of the background scalar field which is coupled to the fermions, is imagined to evolve continuously and slowly from a topologically trivial configuration. With the aid of their adiabatic method, they concluded that when the system does not possess CP symmetry, the fermion number of the soliton can be any real value, not just $\pm \frac{1}{2}$. Later on the computational technique of the adiabatic method was modified by MacKenzie and Wilczek, such that the requirement of adiabaticity was lifted. In their method one computes the energy spectrum of the fermion in the presence of a prescribed soliton and deduces the induced vacuum polarization. They used it to illustrate the vacuum polarization of fermion fields by solitons [38, and then applied it for the case of infinitely sharp soliton as an example [39]. Using this method, the vacuum polarization by solitons for an exactly solvable model was computed by Gousheh and Mobilia [40. The model was general enough to include both the adiabatic and non-adiabatic cases, and they showed that even in the nonadiabatic cases solitons can polarize the vacuum. Only the infinitely sharp solitons can never polarize the vacuum.

The simplest example of a static topological solitary wave in $(1+1)$ dimensions is the kink of the $\phi^{4}$ model which has many applications in quantum field theory [41], condensed-matter physics [42, and cosmology [43. It is worth mentioning that since in the literature the distinction between solitary waves and solitons is not highlighted and they are used interchangeably, we will do the same in this paper. The model we investigate is a $(1+1)$-dimensional theory in which a pseudoscalar field within the non-linear sigma model is coupled to a fermion. The value of the pseudoscalar field at $x= \pm \infty$, denoted by $\pm \theta_{0}$, is a free parameter in the model. However, we are eventually interested in a model in which the pseudoscalar field is a solitary wave, i.e. we let $\theta_{0}$ evolve from 0 to $\pi$, with apriori undetermined shape. Although, many aspects of this model, including the quantum corrections, have been investigated in the literature, many important issues at the zero order are yet to be investigated. The purpose of this paper is to precisely do that. We solve the classical field equations of this theory exactly, within numerical approximation, and extract many important physical implications. The numerical method that 
we use is the relaxation method. We also compare our results with two interesting models. One of them is the exactly solvable model presented by Gousheh and Mobilia [40, in which a prescribed piecewise linear pseudoscalar field with arbitrary boundary conditions has been used and the complete fermionic eigenfunctions and eigenvalues have been obtained. The shape of the pseudoscalar field is chosen so as to qualitatively approximate the isolated kink. The second one is similar to the first, except for the replacement of the piecewise linear field by the isolated kink solution as a prescribed background field. The latter is not exactly solvable and we solve it by using the same numerical method as our model and, as expected, its results are very similar to our model. We find that the exact (relaxed) shape of the soliton in our model is very close to that of the isolated kink. However, the spectrum of the fermion is completely altered due to the presence of the interaction with the background field. We also find that the total energy in the relaxed model is lower than the exactly solvable model with the prescribed soliton. Our solutions indicate that when the mass of the fermion $M$ and soliton are equal, the mass of the elementary boson is $m \approx 0.116 M$, thus giving a strong indication for the validity of the Skyrme's phenomenological model. We also find the surprising result that the true (relaxed) shape of the soliton depends slightly on the fermionic level considered. Moreover, comparison of the exact results of our system with those of the system with the prescribed kink, shows that the latter is a very good approximation for the former. In section 2 we introduce the coupled fermion-soliton model, find the dynamical equations, discuss the symmetries of the system and how they enter the boundary conditions. We also discuss very briefly the numerical method used. In section 3 we discuss the results, including the pattern of the fermion bound state energies and wave functions, the shape of the solitons, and the total energies for the three models.

\section{The model of a coupled fermion-soliton system}

Consider the following Lagrangian describing a fermion interacting non-linearly with a pseudoscalar field $\phi(x, t)$ in $(1+1)$ dimensions:

$$
\begin{aligned}
\mathcal{L} & =\bar{\psi}(x, t)\left[i \gamma^{\mu} \partial_{\mu}-M e^{i \phi(x, t) \gamma^{5}}\right] \psi(x, t) \\
& -\frac{1}{4} \lambda\left[\phi^{2}(x, t)-\frac{m^{2}}{\lambda}\right]^{2}+\frac{1}{2} \partial_{\mu} \phi(x, t) \partial^{\mu} \phi(x, t) .
\end{aligned}
$$

Here $\mu=0,1$ and the parameters $M$ and $m$ denote the masses of the fermionic and bosonic fields, respectively. We choose the following representation for the Dirac matrices: $\gamma^{0}=\sigma_{1}, \gamma^{1}=i \sigma_{3}$, $\gamma^{5}=\gamma^{0} \gamma^{1}=\sigma_{2}$. Then the equations of motion become

$$
i \sigma_{1} \partial_{t} \psi-\sigma_{3} \partial_{x} \psi-M\left[\cos \phi(x, t)+i \sigma_{2} \sin \phi(x, t)\right] \psi=0,
$$

and

$$
\partial_{t} \partial^{t} \phi(x, t)+\partial_{x} \partial^{x} \phi(x, t)+i M \bar{\psi} \sigma_{2} e^{i \phi(x, t) \sigma_{2}} \psi+\lambda \phi^{3}(x, t)-m^{2} \phi(x, t)=0,
$$

where

$$
\psi=\left(\begin{array}{c}
\psi_{1} \\
\psi_{2}
\end{array}\right)
$$

Let us define

$$
\xi=e^{-i E t}\left(\begin{array}{c}
\xi_{1}+i \xi_{2} \\
\xi_{3}+i \xi_{4}
\end{array}\right)=\left(\begin{array}{c}
\psi_{1}+i \psi_{2} \\
\psi_{1}-i \psi_{2}
\end{array}\right)
$$


We are interested in the solitary wave solution of the $\phi^{4}$ theory, in which $\partial_{t} \phi=0$. Inserting Eq. (51) into Eqs. (2) and (3) and using $\partial_{t} \phi=0$, we obtain

$$
\begin{gathered}
-\partial_{x} \partial_{x} \phi(x)-M \cos \phi(x)\left(\xi_{1} \xi_{3}+\xi_{2} \xi_{4}\right) \\
+M \sin \phi(x)\left(\xi_{1} \xi_{4}-\xi_{2} \xi_{3}\right)+\lambda \phi^{3}(x)-m^{2} \phi(x)=0, \\
\xi_{1}^{\prime}+M \cos \phi(x) \xi_{3}-E \xi_{2}-M \sin \phi(x) \xi_{4}=0, \\
\xi_{2}^{\prime}+M \cos \phi(x) \xi_{4}+E \xi_{1}+M \sin \phi(x) \xi_{3}=0, \\
\xi_{3}^{\prime}+M \cos \phi(x) \xi_{1}+E \xi_{4}+M \sin \phi(x) \xi_{2}=0, \\
\xi_{4}^{\prime}+M \cos \phi(x) \xi_{2}-E \xi_{3}-M \sin \phi(x) \xi_{1}=0,
\end{gathered}
$$

where prime denotes differentiation with respect to $x$.

This set of equations cannot be solved analytically. Therefore, we use a numerical method called the relaxation method which determines the solution by starting with a guess and improving it iteratively to relax to the true solution [44. In order to have a rapid convergence, we need a good initial guess which is close to the true solutions and it also should satisfy the same boundary solitons as the real solutions. Thus, we choose the fermionic bound states of the system solved by Gousheh and Mobilia [40] as the initial trial solution. Rather than a coupled system in which both $\psi$ and $\phi$ are treated as dynamical fields, they studied the spectrum of the fermion in the presence of a prescribed $\phi(x)$. They considered an interacting system of a fermion and a pseudoscalar field $\phi(x)$ described by the Lagrangian

$$
\mathcal{L}=\bar{\psi}\left(i \gamma^{\mu} \partial_{\mu}-M e^{i \phi(x) \gamma^{5}}\right) \psi
$$

They chose $\phi(x)$ to be piecewise linear as shown in Fig. (11) by the dashed line. This is qualitatively similar to the kink of the $\phi^{4}$ theory (dotted line in (10) or the soliton of the sine-Gordon theory. The eigenfunctions of the Hamiltonian, i.e. the solutions to the equations (6610), can be chosen to be also the eigenfunctions of the parity operator since the Hamiltonian is invariant under parity. In the previous representation the parity operation on the fermionic field is given by $P \psi(x, t)=\sigma_{1} \psi(-x, t)$. In the representation (5), it becomes $P \xi(x, t)=-\sigma_{2} \xi(-x, t)$. Since we shall choose our solutions to be eigenstates of the parity operator, it is sufficient to solve the equations only for $x \geq 0$. Also, we map the $x$-interval $[0, \infty)$ to $[0,1]$ by $X=\tanh (x)$. In the language of the relaxation method there are seven coupled first-order ODEs: one secondorder, four first-order equations and one equation for the eigenvalue which is the fermion energy. Therefore, we need seven conditions at two boundaries $X=0$ and $X=1$. At $X=0$ we choose three conditions: one parity condition, $\phi(0)=0$ and one giving a value to one of the $\xi_{i}(0) \mathrm{s}$. This value is allowed to change so as to normalized $\psi(x)$. This is extremely important since the equation for $\phi(x)$ is non-linear both in $\phi$ and $\psi$. At $X=1$ we choose four conditions: three of the $\xi_{i}(1)$ s are set to zero and $\phi(1)=\theta_{0}$ (in most cases we set $\theta_{0}=\pi$, in order to have a soliton with winding number one). In addition to these boundary conditions, we have to add another condition to obtain a proper solitonic solution for $\phi(x)$, i.e. one with finite energy and localized energy density. Since in our model $U(\phi)=\frac{1}{4} \lambda\left(\phi^{2}(x, t)-\frac{m^{2}}{\lambda}\right)^{2}$, we have to set $\theta_{0}=\frac{m}{\sqrt{\lambda}}$ in all cases in order to obtain a solitonic solution for $\phi(x)$. 


\section{Results}

To simplify the numerical calculations, we rescale the quantities of the system by dividing Eq. (2) by $M^{3 / 2}$ and Eq. (3) by $M^{2}$. Thus, the scaled equations of motion are in the following form

$$
i \sigma_{1} \partial_{t^{\prime}} \psi^{\prime}-\sigma_{3} \partial_{x^{\prime}} \psi^{\prime}-\left[\cos \phi^{\prime}\left(x^{\prime}, t^{\prime}\right)+i \sigma_{2} \sin \phi^{\prime}\left(x^{\prime}, t^{\prime}\right)\right] \psi^{\prime}=0,
$$

and

$$
\begin{aligned}
\partial_{t^{\prime}} \partial^{t^{\prime}} \phi^{\prime}\left(x^{\prime}, t^{\prime}\right) & +\partial_{x^{\prime}} \partial^{x^{\prime}} \phi^{\prime}\left(x^{\prime}, t^{\prime}\right)+i \bar{\psi}^{\prime} \sigma_{2} e^{i \phi^{\prime}\left(x^{\prime}, t^{\prime}\right) \sigma_{2}} \psi^{\prime} \\
& +\lambda^{\prime} \phi^{\prime 3}\left(x^{\prime}, t^{\prime}\right)-m^{\prime 2} \phi^{\prime}\left(x^{\prime}, t^{\prime}\right)=0 .
\end{aligned}
$$

Now all of the quantities are dimensionless and are given by $t^{\prime}=M t, x^{\prime}=M x, \psi^{\prime}=\frac{\psi}{\sqrt{M}}, \phi^{\prime}=\phi$, $m^{\prime}=\frac{m}{M}$ and $\lambda^{\prime}=\frac{\lambda}{M^{2}}$. From now on we drop the primes for simplicity. In most of the results presented here, we compare three models. The first model which we call model I is our model, i.e. an eigenvalue problem for a fermion chirally coupled to a pseudoscalar field which is not prescribed and its shape, along with the functional forms of the fermionic states, are determined by the equations of motion. The second model which we refer to as model II is similar to our model, except that in this model the pseudoscalar field coupled to fermion is precisely the kink as a prescribed background field and cannot be altered by the presence of the fermion. The third one which we refer to as model III is the exactly solvable model solved by Gousheh and Mobilia, in which again the pseudoscalar field is prescribed but in the form of a piecewise linear field.

In Fig. (1) we show $\phi(x)$ for $m=1$ and $\theta_{0}=\pi$ for three cases: the one obtained dynamically for our system (solid line), the prescribed kink solution (dotted line) and the prescribed form chosen in [40] (dashed line). It can be seen that the solitonic solution is affected very slightly by the fermion; the soliton in the presence of the fermion is so similar to the isolated kink that they cannot be easily distinguished from each other. Therefore, the back reaction of the fermion on the soliton is indeed very small.

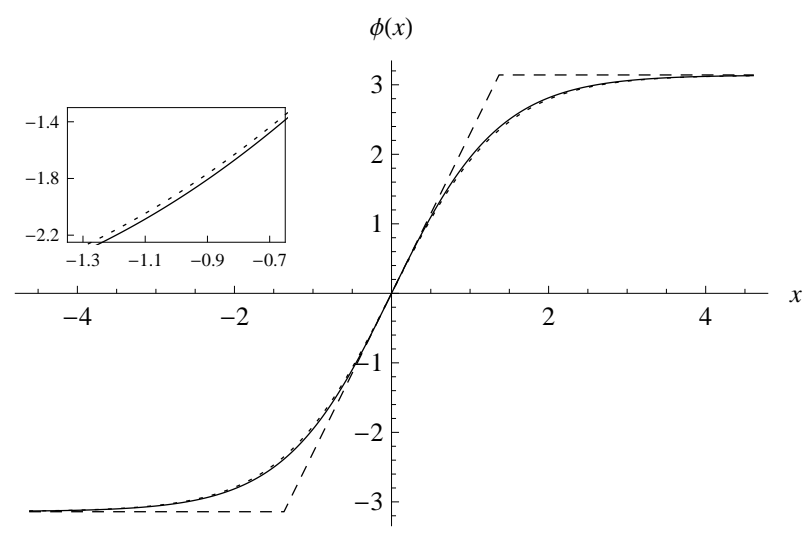

Figure 1: The graphs of the $\phi(x)$ for three cases: solid line depicts the $\phi(x)$ obtained from our model (model I), dotted line the prescribed kink solution of the pure $\phi^{4}$ theory (model II), and dashed line the prescribed background field of the exactly solvable model (model III).

In Fig. (2) we show the fermionic bound state energies in the interval $0 \leqslant \theta_{0} \leqslant 2 \pi$ for the model I (solid lines), the model II (dashed lines) and the model III (dotdashed lines). In this figure the slope of the pseudoscalar field at $x=0$ is fixed at about 2.957 for all three models. This slope is important in model III, where the critical point of a bound state crossing $E=0$ 
occurs at $\theta_{0}=\pi$. In the models I and II this crossing would happen for a greater value of $\theta_{0}$. Therefore, the domain of the adiabatic region is larger in the relaxed state and the isolated kink which is similar to it. This result can also be illustrated as follows. If we insist that the energy level crossing occurs at $\theta_{0}=\pi$ in all cases, the slope of the soliton with winding number one would be greater in models I and II. Therefore, the domain of the adiabatic region as measured by the slope is larger. Comparison between the energies of the models I and II shows that choosing kink as a prescribed background is a very good approximation for our system.

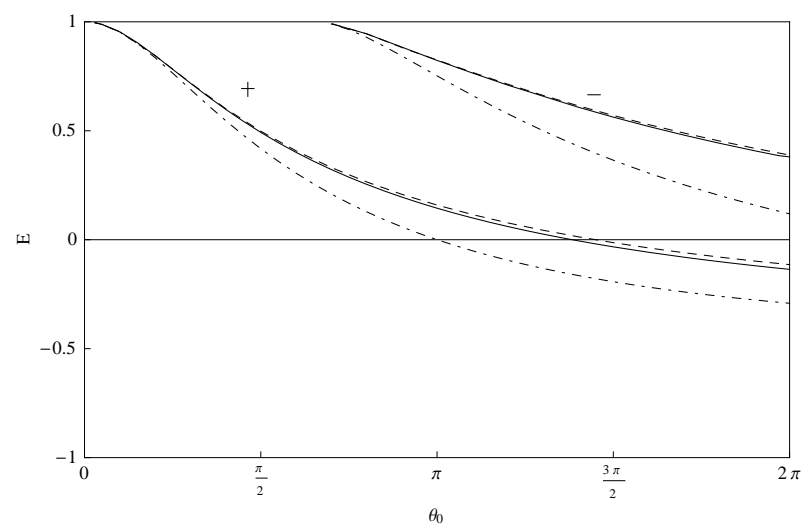

Figure 2: The fermionic bound state energies when the slope of the pseudoscalar fields at $x=0$ is $\mu \approx 2.957$, in the interval $0 \leqslant \theta_{0} \leqslant 2 \pi$. Solid, dashed and dotdashed lines depict the fermion energies for the models I, II and III, respectively. The plus and minus signs indicate the parity of the bound states.

From now on we set $\theta_{0}=\pi$ in all cases. Figure (3) shows $\xi_{i}$ s for the lowest bound state for the case $m=1$. Solid lines refer to the results obtained numerically for our lagrangian (model I) and dashed lines refer to the solutions of the equations of the Lagrangian (11) in which $\phi(x)$ is the piecewise linear form shown by the dashed line in Fig. (1) (model III). Since there are very small and indistinguishable differences between the solutions of the models I and II, we do not present the results of the model II.

Figure (5) shows the energy of the lowest bound state of the fermion as a function of the boson mass. In this graph we also show the fermion energy of the model II. Note that, as expected, the two energies are close and the relaxed one (model I) is slightly lower. The total boson energy, sometimes called the classical soliton mass $M_{c l}$, as a function of the boson mass is presented in Fig. (6). Note that for an isolated kink, the functional form would be exactly linear as given by $M_{c l}=\frac{2 \sqrt{2}}{3} \theta_{0}^{2} m$, as depicted in the figure by the solid line. In this figure the result of the model I is shown by the points to make these two results (models I and II) distinguishable. It is interesting to note that the two results are too close to each other to be distinguished. We can quantify the difference between the functional forms of the relaxed and the isolated kink by computing their point-wise root mean squared difference. We have plotted this quantity as a function of the energy of the lowest fermionic bound state which is coupled to the soliton in Fig. (44). As it is evident from the graph, when mass of the elementary boson and therefore the soliton is infinite, the shape of the soliton is completely unaffected by the presence of the fermion and $\delta_{r m s}(\phi) \rightarrow 0$. As the mass of the boson is decreased, its sensitivity to the presence of the fermion increases. An important point is worth mentioning. It has been discovered as early as 1976 [32, 45, 46, that when the problem possesses particle conjugation symmetry, the zero fermionic mode has 

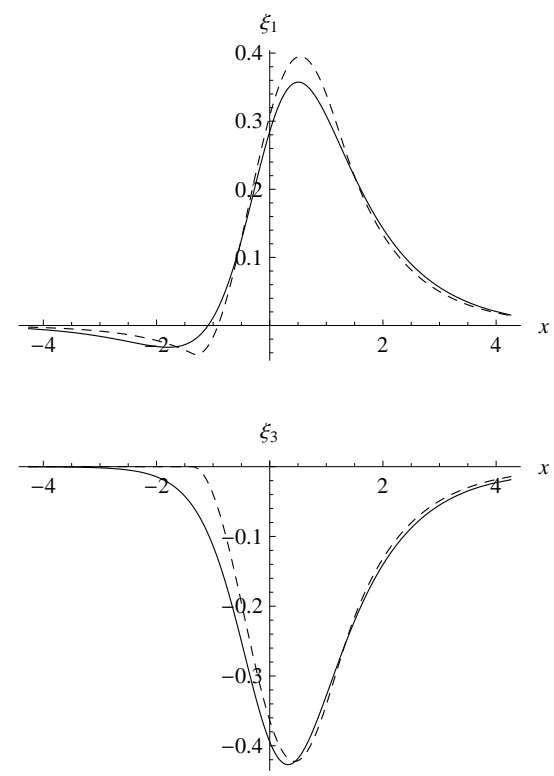
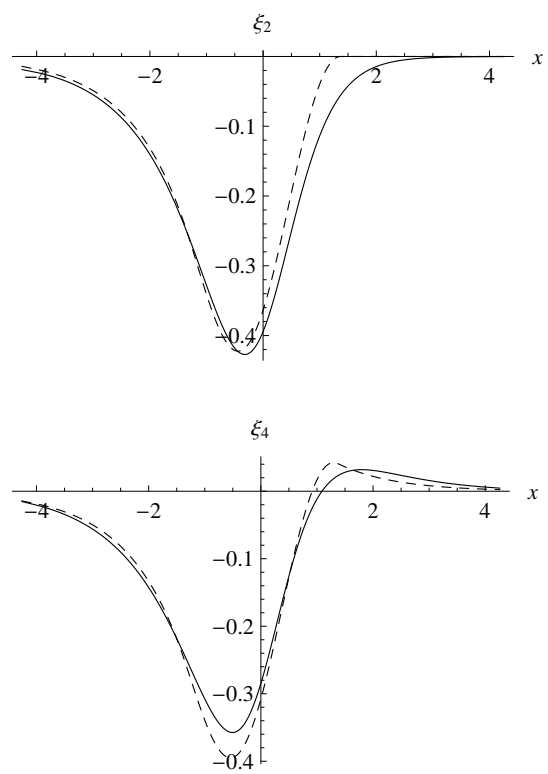

Figure 3: The lowest bound state of the fermion as a function of $x$. Solid curves are the solutions to model I and dashed lines the solutions to the model III. The parity is positive and both the fermion and the boson have unit masses.

absolutely no back reaction on the soliton. In fact one can show on general grounds (using the definition of this operator, its unitarity property and general properties of the $\gamma$-matrices) that when the problem possesses this symmetry, the zero modes produce no back reaction. However, our problem does not have this symmetry and the effect of the zero mode on the shape of the soliton is non-zero. As a matter of fact Fig. (41) shows that the pattern of $\delta_{r m s}(\phi)$ does not show any irregularity at $e_{f}=0$.

In the Skyrme model [3] the pions are introduced as the pseudoscalar background field, coupled to baryons playing the role of fermions. Skyrme conjectured that the solitonic excitations of pions, later on called skyrmions, are indeed nucleons. This conjecture has been studied by many authors and used as an approximate phenomenological model at energy scales up to about a few Gev (see for example [47, 48, 49, 50, 51]). Our results are also consistent with this conjecture. By extrapolating the results displayed in Fig. (66) for our model, one can conclude that when $M_{c l}=1$, the elementary boson mass is $m \approx 0.116$, all in units of the fermion mass $M$. For the soliton with $M_{c l}=1, \mu \approx 0.258$ which corresponds to a wide soliton with winding number one. Also, as can be seen from Fig. (5), at $m \approx 0.116$ there is no fermion energy level crossing zero. Therefore, here the soliton of mass $M_{c l}=1$ polarizes the vacuum and its ground state has fermion number one. The pion mass is about $0.14 \mathrm{Gev}$ and the nucleon mass is about 1.0Gev. Therefore, if in our model the pseudoscalar field $\phi(x)$ plays the role of the pions, solitonic excitations (with $M_{c l}=1$ for $m \approx 0.116$ ) could be considered to be nucleons. Note that in the Skyrme model there are three pions introduced as in a non-linear sigma model and two doublets for two nucleons, but in our model there are only one real scalar field and one fermion field. This could partly account for the difference in the results.

Figure (7) shows the vacuum polarization induced by the soliton as a function of $\theta_{0}$ when the slope of the pseudoscalar field at $x=0$ is $\mu \approx 2.957$. Solid lines show the result of our model (model I) in which the fermion energy level crosses $E=0$ at $\theta_{0} \approx 1.38 \pi$ and the dashed lines show the result of the model III in which the fermion energy level crosses $E=0$ at $\theta_{0}=\pi$. 


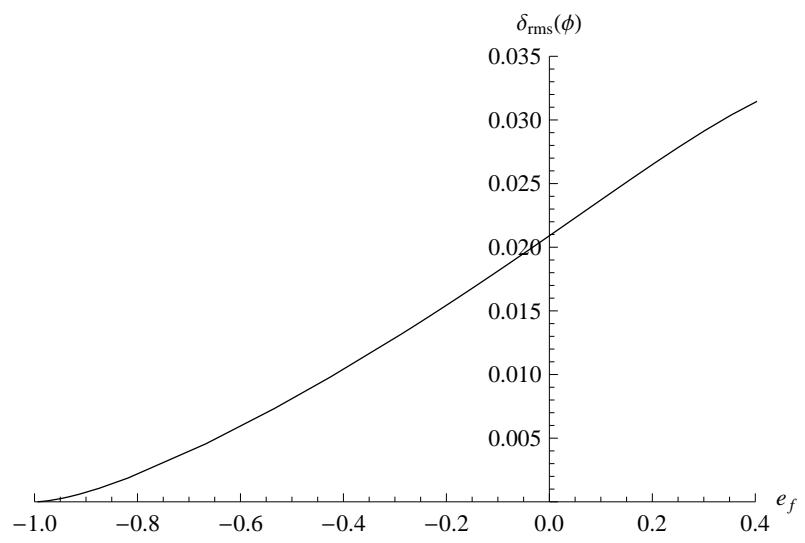

Figure 4: This is a plot of the point-wise root mean squared difference between the relaxed and the isolated kink. The independent variable is the energy of the fermionic bound state which is coupled to the soliton and is chosen to be the lowest bound state. This axis could equally have been chosen to be the mass of the elementary boson $(m)$. The correspondence between the two is that $e_{f}=-1(M) \leftrightarrow m=\infty$ and $e_{f}=0.4(M) \leftrightarrow m \approx 0.8(M)$. The numerical results for $\delta_{r m s}(\phi)$ becomes unreliable, when mass of the elementary boson is smaller than $0.6(M)$.

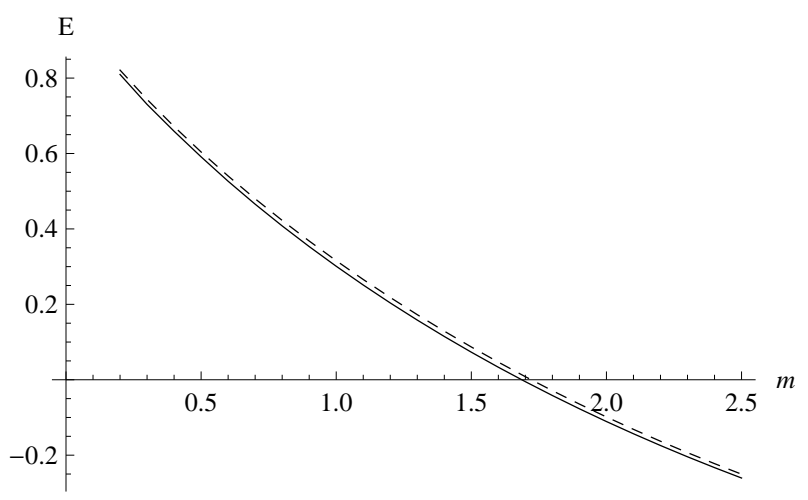

Figure 5: The energy of the lowest bound state of the fermion as a function of the boson mass for the model I (solid line) and model II (dashed line) at $\theta_{0}=\pi$.

There are two contributions to the vacuum polarization. First is the adiabatic contribution, predicted by Goldstone and Wilzcek [37, which is a linear decrease with slope $-\theta_{0} / \pi$ whenever the interaction is in the form of $\bar{\psi} e^{i \phi \gamma^{5}} \psi$. This is due to the occurrence of the spectral deficiency in the Dirac sea as $\theta_{0}$ increases. The second is the non-adiabatic contribution which occurs only when a fermionic energy level crosses $E=0$. In the latter case there is a jump of +1 in the vacuum polarization since this energy level is filled after crossing $E=0$ in the vacuum state, by definition. These two contributions have been explicitly derived for model III in reference [40]. There is a potential ambiguity of whether the state at $E=0$ is to be considered filled or empty in the vacuum state. If this is considered as a true ambiguity, one can resolve it by observing that the vacuum polarization has to be zero at $\theta_{0}=0$, and self-consistency and symmetry dictates the situation at the other points as shown in Fig. (7). We should mention that in our model which is partially based on $\lambda \phi^{4}$ theory, we have only two distinct topological sectors: the trivial and the topological charge \pm 1 sectors. However, the fermion responds to the value of $\theta_{0}$, regardless of the topological charge.

In Fig. (8), we show the total energy, i.e. the lowest fermionic bound state energy plus the 


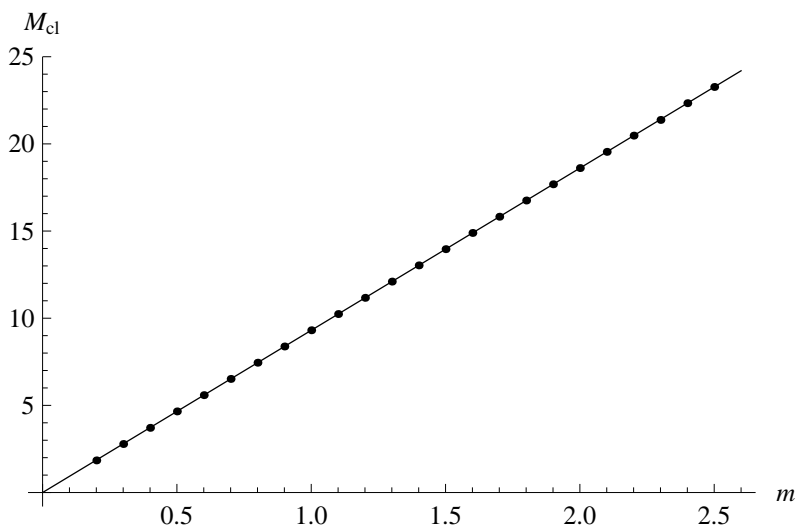

Figure 6: The total energy of the soliton as a function of the boson mass. The points show the numerical results for the model I and solid line shows the kink mass which behaves as $M_{c l}=\frac{2 \sqrt{2}}{3} \theta_{0}^{2} m$.

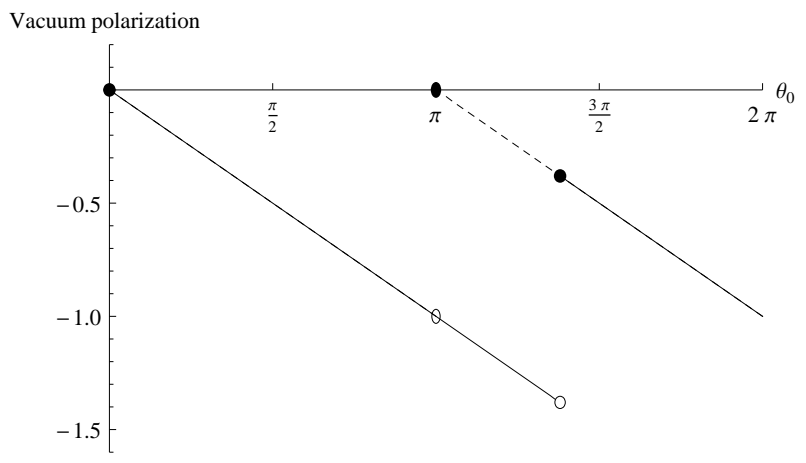

Figure 7: The vacuum polarization by the soliton as a function of $\theta_{0}$ when the slope of the pseudoscalar field at $x=0$ is $\mu \approx 2.957$. The solid lines show the result for our model and the dashed lines the result for the model III. The filled and empty circles at $\theta_{0} \approx 1.38 \pi$ are for model I and the filled and empty ellipses at $\theta_{0}=\pi$ are for model III.

soliton energy for both models I and III. After obtaining the solution of our model for each value of $m$, we set the slope $\mu$ of model III to the slope of $\phi(x)$ obtained at $x=0$. In this way we can compare the energies of two models for each $m$. As expected, the total energy for the relaxed kink (model I) is lower than for the model III.

In Fig. (9) we compare $\phi(x)$ for two cases: The soliton relaxed with the ground state and the first excited state in which the parities are positive and negative, respectively. The parameters of the system are the same for both cases: $m=1$ and $\theta_{0}=\pi$. As can be seen from the graph, the relaxed solitons of these two states are not the same.

\section{Conclusion}

In this paper, we have considered a fermion field chirally coupled to a pseudoscalar field which could evolve into a soliton in $(1+1)$ dimensions. We have solved the system self-consistently and found the lowest lying bound states of the fermion and the shape of the soliton non-perturbatively and exactly to within our numerical approximation. We have also presented the results of a similar model in which the pseudoscalar field is the prescribed kink instead of a field which can be altered due to the presence of the fermion. Comparing the results of this model, including those of the 


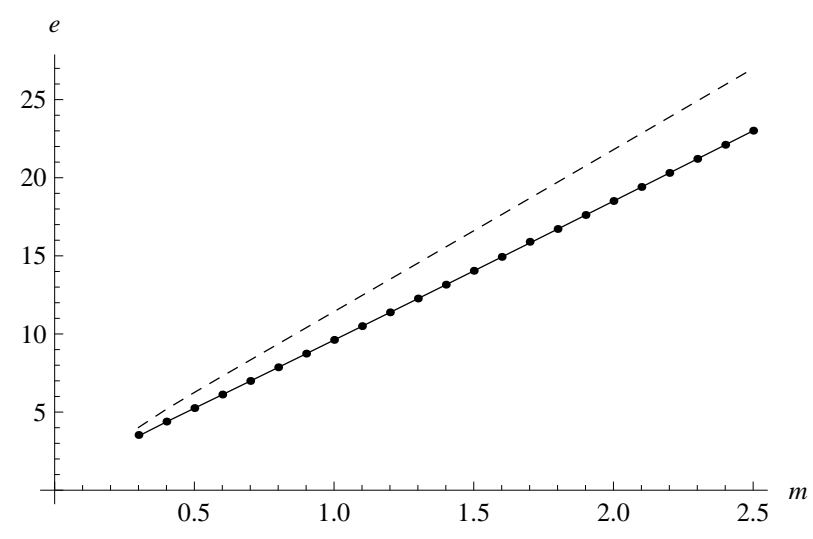

Figure 8: The total energy (the lowest fermionic bound state energy plus the soliton energy) as a function of the boson mass. Dashed line represents the total energy for the model III, and solid line represents the total energy for the model II and the points the total energy for our model.

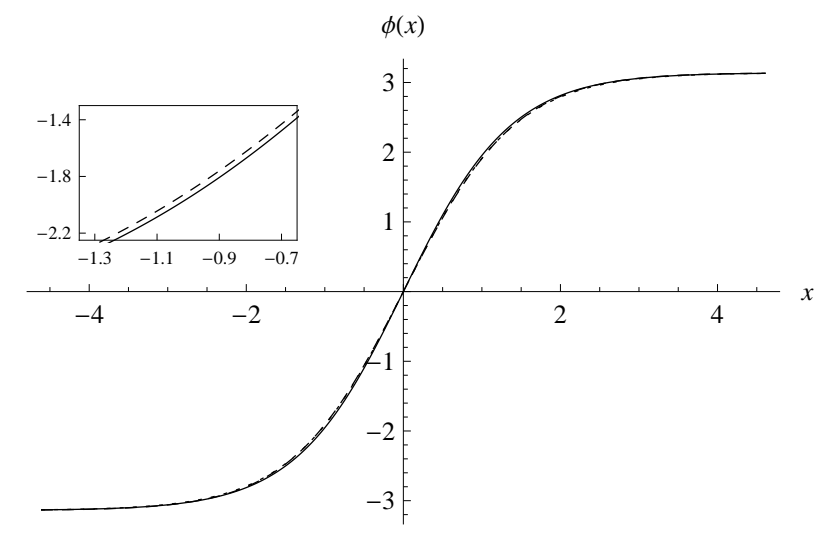

Figure 9: $\phi(x)$ for two cases: solid line shows the soliton relaxed along with the lowest fermionic bound state and dashed line shows the soliton relaxed along with the first excited state.

fermionic states, with our system, we find that we can approximate the exact (relaxed) kink by the isolated kink, as is usually done. We have shown that in the models with the relaxed kink and the prescribed kink, the adiabatic region is larger than the model with the prescribed piecewise linear background field as a soliton. We have also computed the vacuum polarization of the fermion induced by the presence of the soliton and displayed the results for our model and the exactly solvable prescribed model. In this connection we have elaborated on the adiabatic and non-adiabatic contributions to the vacuum polarization. In addition, we have determined that when the soliton mass $M_{c l}$ is equal to the fermion mass $M$, the elementary boson mass $m \approx 0.116 M$ which is in a good agreement with the conjecture of Skyrme. Moreover, we have shown that the relaxed form of the soliton depends on the fermionic state to which it is coupled. It is worth mentioning that we have not considered the back reaction perturbatively but exactly to within the numerical approximation.

\section{References}

[1] T.H.R. Skyrme, A non-linear theory of strong interactions, Proc. Roy. Soc. London A 247, 260 (1958). 
[2] T.H.R. Skyrme, A unified model of $K$ - and $\pi$-mesons, Proc. Roy. Soc. London A 252, 236 (1959).

[3] T.H.R. Skyrme, A non-linear field theory, Proc. Roy. Soc. London A 260, 127 (1961).

[4] T.H.R. Skyrme, A unified field theory of mesons and baryons, Nucl. Phys. 31, 556 (1962).

[5] T.H.R. Skyrme, Kinks and the Dirac equation, J. Math. Phys. 121735 (1971).

[6] A. Chodos, R.L. Jaffe, K. Johnson, C.B. Thorn and V.F. Weisskopf, A new extended model of hadrons, Phys. Rev. D 9, 3471 (1974).

[7] M. Creutz, Quark bags and local field theory, Phys. Rev. D 10, 1749 (1974).

[8] T.A. DeGrand, R.L. Jaffe, K. Johnson and J.E. Kiskis, Masses and other parameters of the light hadrons, Phys. Rev. D 12, 2060 (1975).

[9] W.A. Bardeen, M.S. Chanowitz, S.D. Drell, M. Weinstein and T.-M. Yan, Heavy quarks and strong binding: a field theory of hadron structure, Phys. Rev. D 11, 1094 (1975).

[10] C. Rebbi, Nonspherical deformations of hadronic bags, Phys. Rev. D 12, 2407 (1975).

[11] V. Vento, M. Rho, E.M. Nyman, J.H. Jun and G.E. Brown, Chiral pion dynamics for spherical nucleon bags, Nucl. Phys. A 345, 413 (1980).

[12] F. Myhrer, G.E. Brown and Z. Xu, The baryon masses and the chiral quark bag model, Nucl. Phys. A 362, 317 (1981).

[13] R. Goldflam and L. Wilets, The soliton bag model, Phys. Rev. D 25, 1951 (1982).

[14] R. Friedberg and T.D. Lee, Fermion field nontopological solitons. 1, Phys. Rev. D 15, 1694 (1977).

[15] R. Friedberg and T.D. Lee, Fermion field nontopological solitons. 2. Models for hadrons, Phys. Rev. D 16, 1096 (1977).

[16] J.A. Bagger and S.G. Naculich, Quantum corrections deflate deep bags, Phys. Rev. Lett. 67, $2252(1991)$.

[17] J.A. Bagger and S.G. Naculich, Quantum bags at strong coupling, Phys. Rev. D 45, 1395 (1992).

[18] S.G. Naculich, Quantum kinks: solitons at strong coupling, Phys. Rev. D 46, 5487 (1992).

[19] E. Farhi, N. Graham, R.L. Jaffe and H. Weigel, A heavy fermion can create a soliton: a $1+1$ dimensional example, Phys. Lett. B 475, 335 (2000).

[20] E. Farhi, N. Graham, R.L. Jaffe and H. Weigel, Heavy fermion stabilization of solitons in $1+1$ dimensions, Nucl. Phys. B 585, 443 (2000).

[21] Z. Surujon, Higgs localization in split fermion models, Phys. Rev. D 73, 016008 (2006).

[22] C. Bogdanos, A. Dimitriadis and K. Tamvakis, Brane models with a Ricci-coupled scalar field, Phys. Rev. D 74, 045003 (2006). 
[23] Y.-X. Liu, X.-H. Zhang, L.-D. Zhang and Y.-S. Duan, Localization of matters on pure geometrical thick branes, JHEP 02, 067 (2008).

[24] V.A. Rubakov and M.E. Shaposhnikov, Do we live inside a domain wall?, Phys. Lett. B 125, 136 (1983).

[25] M.V. Libanov and S.V. Troitsky, Three fermionic generations on a topological defect in extra dimensions, Nucl. Phys. B 599, 319 (2001).

[26] J.M. Frere, M.V. Libanov and S.V. Troitsky, Three generations on a local vortex in extra dimensions, Phys. Lett. B 512, 169 (2001).

[27] S. Randjbar-Daemi and M. Shaposhnikov, QED from six-dimensional vortex and gauge anomalies, JHEP 04, 016 (2003).

[28] W. Nahm and D.H. Tchrakian, Localisation of fermions to brane: codimension $d \geq 2$, JHEP 04, 075 (2004).

[29] A. Melfo, N. Pantoja and J.D. Tempo, Fermion localization on thick branes, Phys. Rev. D 73, 044033 (2006).

[30] G. Gibbons, K.-I. Maeda and Y.-I. Takamizu, Fermions on colliding branes, Phys. Lett. B 647, 1 (2007).

[31] X.-H. Zhang, Y.-X. Liu and Y.-S. Duan, Localization of fermionic fields on braneworlds with bulk tachyon matter, Mod. Phys. Lett. A 23, 2093 (2008).

[32] R. Jackiw and C. Rebbi, Solitons with fermion number 1/2, Phys. Rev. D 13, 3398 (1976).

[33] W.P. Su, J.R. Schrieffer and A.J. Heeger, Solitons in polyacetylene, Phys. Rev. Lett. 42, 1698 (1979).

[34] W.P. Su and J.R. Schrieffer, Fractionally charged excitations in charge-density-wave systems with commensurability 3, Phys. Rev. Lett. 46, 738 (1981).

[35] M. Rice and E. Mele, Elementary excitations of a linearly conjugated diatomic polymer, Phys. Rev. Lett. 49, 1455 (1982).

[36] R. Jackiw and G.W. Semenoff, Continuum quantum field theory for a linearly conjugated diatomic polymer with fermion fractionization, Phys. Rev. Lett. 50, 439 (1983).

[37] J. Goldstone and F. Wilczek, Fractional quantum numbers on solitons, Phys. Rev. Lett. 47, 986 (1981).

[38] R. MacKenzie and F. Wilczek, Illustrations of vacuum polarization by solitons, Phys. Rev. D 30, 2194 (1984).

[39] R. MacKenzie and F. Wilczek, Examples of vacuum polarization by solitons, Phys. Rev. D 30, 2260 (1984).

[40] S.S. Gousheh and R. López-Mobilia, Vacuum polarization by solitons in $(1+1)$ dimensions, Nucl. Phys. B 428, 189 (1994).

[41] N. Manton and P. Sutcliffe, Topological Solitons, Cambridge University Press, Cambridge U.K. (2004). 
[42] A.R. Bishop and T. Schneider, Solitons and Condensed Matter Physics, Springer, U.S.A. (1978).

[43] A. Vilenkin and E.P.S. Shellard, Cosmic Strings and Other Topological Defects, Cambridge University Press, Cambridge U.K. (1994).

[44] M.E.J. Newman and G.T. Barkema, Monte Carlo Methods in Statistical Physics, Clarendon Press. Oxford U.K. (2001).

[45] R. Jackiw and P. Rossi, Zero modes of the vortex-fermion system, Nucl. Phys. B 190, 681 (1981).

[46] R. Jackiw and S.-Y. Pi, Chiral gauge theory for graphene, Phys. Rev. Lett. 98, 266402 (2007).

[47] J.G. Williams, Topological analysis of a nonlinear field theory, J. Math. Phys. 11, 2611 (1970).

[48] E. Witten, Current algebra, baryons and quark confinement, Nucl. Phys. B 223, 433 (1983).

[49] G.S. Adkins, C.R. Nappi and E. Witten, Static properties of nucleons in the Skyrme model, Nucl. Phys. B 228, 552 (1983).

[50] G.S. Adkins and C.R. Nappi, The Skyrme model with pion masses, Nucl. Phys. B 233, 109 (1984).

[51] I.J.R. Aitchison, Berry phases, magnetic monopoles and Wess-Zumino terms or how the skyrmion got its spin, Acta Phys. Polon. B 18, 207 (1987). 